\title{
ADAPTIVE FILTER APPLICATIONS TO AUTONOMOUS UNDERWATER VEHICLE
}

\author{
D.G. Lainiotis, Dinesh Menon, K.N. Plataniotis, C.J. Charalampous
}

\author{
Florida Institute of Technology
}

\section{MELBOURNE, FLORIDA}

\begin{abstract}
A powerful adaptive filter algorithm for estimation of forces acting on an Autonomous Underwater Vehicle (AUV), and the corresponding adaptive control law which determines the desired heading commands are considered in this paper. Due to the complicated nature of AUV's task and incorporation of the ocean current, uncertain parameters arise in the equations of motion that constitute the AUV's dynamic model. Based on the Lainiotis Partitioning Theory, a highly adaptive scheme is used to estimate the velocity components, for controlling the vehicle. Extensive simulations demonstrate the robustness and the effectiveness of the new scheme in a variety of different environments.
\end{abstract}

\section{INTRODUCTION}

Autonomous Underwater Vehicles (AUVs) are an important tool for various undersea tasks such as construction [1], repairing of underwater equipment [2], waste management, pollution monitoring, undersea research and data collection [3]. Due to the complicated nature of the tasks and the potential for assuming various payloads, there is a need for tight control and maneuverability of the AUV under large variations in the operating conditions. A robust control algorithm that can enhance the efficiency of the vehicle is therefore highly desirable [2],[3],[4]. However, due to the ocean current and the nature of the vehicle most of the available methods have limited applicability [2],[4].

The dynamics of the autonomous underwater vehicle are nonlinear in nature due to rigid body coupling and the hydrodynamic forces of the vehicle. Moreover most autonomous vehicles perform maneuvers in different directions using thrusters, which makes the uncertainties even more severe. Recently, several control strategies for the AUVs have been discussed. Most of these methods require linearized models. The main problem in these methods is that the robustness of the control scheme with respect to parameter uncertainties cannot be guaranteed, since the linearized set of equations have to be corrected almost continuously, to account for frequent changes in the operating point of the vehicie. Hence an adaptive contol scheme is required that will account for these hydrodynamic uncertainties and variable loading conditions. The proposed strategy is called the Partitioned Adaptive Control. Based on the general theory of Partitioning algorithms, the new method uses the Adaptive Lainiotis Filter for estimation and a quadratic performance criterion, to calculate the required control input [7],,99],[10].

Specifically, the paper is organized as follows: Section II describes the model representing the AUV motion. In Section III the Adaptive Lainiotis approach to estimation and control is discussed. The simulation results and conclusions are presented in Sections IV and $\mathrm{V}$, respectively.

\section{THE AUV MODEL}

Several continuous state-space models have been developed based on the nonlinear equations of motion derived for an underwater vehicle. The most complete set of equations of motion for underwater vehicles are in [5]. These models are basically representations of the vehicle dynamics. The non-linear equations of the underwater motion are by themselves very hard to analyze. In most of the cases, a linearized version of the overall model is used. The linearized model is not capable of simulating the entire movement of the vehicle around a particular axis at any time, but it is valid around one operating point, which is very important, because the underwater vehicle performs tasks which depend on very precise maneuvers.

According to [4], if the cross-coupling in the model is 
ignored, the following linearized model, for rotation around an arbitrary axis can be obtained:

$$
G(s)=\frac{K_{1} \cdot K_{2}}{(T s+1) \cdot(J s+C) \cdot s}
$$

where,

$T=0.3 \mathrm{sec}$. , is the time constant of the propellor $J=2560 \mathrm{lb} . \mathrm{ft}$, is the moment of inertia of the vehicle $C=150$, is the linearized damping coefficient, for rotational motion

The state-space representation of the model developed above is summarized below:

$$
\begin{aligned}
& {\left[\begin{array}{l}
\dot{x}_{1} \\
\dot{x}_{2} \\
\dot{x}_{3}
\end{array}\right]=\left[\begin{array}{ccc}
0 & 1 & 0 \\
0 & 0 & 1 \\
0 & -M & -N
\end{array}\right] \cdot\left[\begin{array}{l}
x_{1} \\
x_{2} \\
x_{3}
\end{array}\right]+\left[\begin{array}{l}
0 \\
0 \\
K
\end{array}\right] \cdot u(t)+w(t)} \\
& z(k)=\left[\begin{array}{lll}
1 & 0 & 0
\end{array}\right] \cdot x(k)+v(k)
\end{aligned}
$$

where,

- $X_{1}, X_{2}$ and $X_{3}$ are the orientation, angular velocity and angular acceleration, respectively, along an arbitrary axis

- $w(t)$ is zero-mean, white, gaussian, plant noise with covariance, $Q$

- $u(t)$ is the control input, in volts

- $z(k)$ are the discrete measurements

- $v(k)$ is zero-mean, white, gaussian, measurement noise with covariance, $R$

The coefficients that describe the model are given by:

$$
\begin{aligned}
& K=\frac{K_{1} \cdot K_{2}}{T \cdot J} \\
& M=\frac{C}{T \cdot J} \\
& N=\frac{(T \cdot C+J)}{T \cdot J}
\end{aligned}
$$

The only information that can be measured from a vehicle for orientation control, are angles. It is obvious from the above definition of the states, that a statistical estimator has to be used in order to provide adequate information to the controller, since the last has to use angular velocities and accelerations as well. Most of the current control schemes use statistical estimators like Kalman filter to obtain the required information. However, the Kalman filter is not the optimal estimator when parametric uncertainty exists. Unfortunately this is exactly the case for an underwater vehicle. It is well known that the condition of the manipulator arm connected to the AUV can significantly affect the moment of inertia of the vehicle depending on its size and degrees of freedom. This can degrade the performance of the controller, since it is obvious from Eqns. (1) and (2) that the above changes in moment of inertia can affect the parameters of the model [ 3], [4].

\section{ADAPTIVE PARTITIONING CONTROL}

It is unrealistic to expect the designer of the controller to know in advance the exact values of the parameters in the model, at any time. However if parametric uncertainty exists, adaptive estimation techniques [6], [10], can be used to provide accurate estimates of the system state. The adaptive estimation/control problem, can be specified as follows:

$$
\begin{gathered}
x(t+1)=\phi\left(t+1, t ; \theta_{i}\right) \times(t)+G\left(t ; \theta_{i}\right) u(t)+w(t) \\
x(t)=H\left(t ; \theta_{i}\right) x(t)+v(t)
\end{gathered}
$$

The above state-space model, has all quantities as described previously. ' $\theta$ ' represents the unknown parameter vector. Using the measurement set, $x(k)=\{z(1), z(2), \ldots, z(L)\}$, the optimal estimate of the system state in the minimum mean square error sense is obtained. The desired control sequence, $\{u(0), u(1), \ldots, u(N-1)\}$, is obtained by minimizing the following quadratic cost criterion:

$$
J=E\left\{x^{T}(N) \cdot C \cdot x(N)+\sum\left[x^{T}(t) \cdot A(t) \cdot x(t)+u^{T} \cdot B(t) \cdot u(t)\right\}\right\}
$$

The solution to the above problem has been addressed by Lainiotis [7], [8], [10]. The details of the approach are summarized below. The adaptive control signal is given by:

$$
\hat{u}(t)=\sum_{i} u^{\prime}\left(t / \theta_{i}\right) p\left(\theta_{i} / t, 0\right)
$$

where,

$\mathbf{u}^{\prime}\left(\mathrm{t} / \theta_{\mathrm{i}}\right)$ is the discrete linear separation control corresponding to model parameter vector, $\theta_{i}$ $p\left(\theta_{i} / t, 0\right)$ is the a-posteriori probability density function which is given by a recursive Baye's rule formula:

$$
p\left(\theta_{i} / L, 0\right)=\frac{L\left(L, 0 / \theta_{i}\right)}{\sum L_{i}\left(L, 0 / \theta_{i}\right) \cdot p\left(\theta_{i} / t-1,0\right)} \cdot p\left(\theta_{i} / t-1,0\right)
$$


where,

$\left.u\left(1,0 / \theta_{i}\right)=\mid P_{2}\left(1 / t-1,0 ; \theta_{i}\right)\right\}^{-\frac{1}{2}} \cdot \exp \left\{-\frac{1}{2}\left|\bar{z}\left(1 / t-1,0 ; \theta_{i}\right) \|\right|_{P_{r}^{-1}\left(1 / t-1,0 ; \theta_{i}\right)}^{2}\right\}$

$\bar{z}\left(t / t-1,0 ; \theta_{i}\right)=2(t) \cdots H\left(t, \theta_{i}\right) \cdot 2\left(t / t-1,0 ; \theta_{i}\right)$

$P_{f}\left(t / t-1,0_{;} \theta_{i}\right)=H\left(t / \theta_{i}\right) \cdot P\left(t / t-1,0_{;} \theta_{i}\right) \cdot u^{T}\left(u / \theta_{i}\right)+R(t)$

$\varepsilon\left(t / t-1,0_{i} \theta_{i}\right)$ is the model conditional Kalman Filter estimate. The PAC structure is given in Fig. 1.

\section{Comments:}

- The effectiveness of the controller relies heavily on the performance of the adaptive estimator. It is well known that the adaptive estimator recognizes the correct model, if the true value of the parameter is present in the sample space, and if it is not, converges to a value that is closest to the true value.

- The partitioning realization has a natural decoupled structure. All the subunits needed to implement the adaptive scheme can be realized independently. In this way, the scheme

-can be implemented on a parallel processing machine, resulting in enormous computational savings [6],[8].

-the overall realization is robust with respect to failure of any of the parallel processors [6],[10].

\section{SIMULATION RESULTS}

The moment of inertia, $J$ is chosen as the unknown parameter vector, $\theta$. A four-fold change in the value of the moment of inertia is assumed and the parameter vector is quantized for values of ' $J$ ' in that range. It is assumed that the actual model parameter value lies in that range. Monte Carlo simulation is carried out using a discrete model with a sampling interval of $0.1 \mathrm{sec}$. The plant and measurement noise covariances, $Q$ and $R$ are assumed to be 0.25 and 0.1 , respectively.

The weighting matrices used for the control law are:

$$
C=\left[\begin{array}{ccc}
50 & 0 & 0 \\
0 & 50 & 0 \\
0 & 0 & 50
\end{array}\right], A=\left[\begin{array}{lll}
1 & 0 & 0 \\
0 & 1 & 0 \\
0 & 0 & 1
\end{array}\right], B=\left[\begin{array}{lll}
1 & 0 & 0 \\
0 & 1 & 0 \\
0 & 0 & 1
\end{array}\right]
$$

Two experiments are conducted:

\section{SIMULATION I:}

In this experiment, four values of the moment of inertia, $J, 2 J, 3 J$ and $4 J$ are used. A bank of linear filters corresponding to each parameter value is implemented. A discrete LQG control law is applied to each model, to calculate the corresponding control gains. The actual model value is assumed to be ' $\mathrm{J}$ '. The adaptive control signal obtained as described in the previous section and the associated mean square error plots are shown in Figs. 2 and 3. The adaptive control signal shows an initial offset from the desired control trajectory, but as the estimation proceeds and the probabilistic weighting gets updated, the adaptive signal exactly follows the desired trajectory. In Fig. 4 the non-linear decision mechanism of the adaptive filter is illustrated. The filter has the ability to detect the correct model in a limited number of steps. The orientation of the AUV along an arbitrary axis and the associated mean square error plots are shown in Figs. 5 and 6. The errors are averaged over 100 Monte Carlo runs using the following criterion:

$$
M S E=\frac{1}{m c} \cdot \sum_{i=1}^{m c}[x(k)-\hat{x}(k / k)]^{2}
$$

\section{SIMULATION II:}

To demonstrate the robustness of the control algorithm, it is assumed that the moment of inertia undergoes a four-fold change after 30 seconds. Fig. 7 shows how the model selection occurs. The control trajectories and the associated mean square error plots are shown in Figs. 8 and 9. Figs. 10 and 11 give the orientation of the vehicle and mean square error between the desired and adaptive esimates.

\section{CONCLUSIONS}

In summary, an adaptive control structure like that of the Partitioned Adaptive Control, can handle parametric uncertainty and provide more meaningful estimates of the states and the control signals. The separation controls are given by the product of a deterministic control gain, which can be computed off-line, and a mean square error causal estimate of the current state. The Kalman filter gains which are not dependent on the measurements can also be computed off-line. All these contribute to an attractive structural property. The emphasis on parallel processing capabilities in the new control designs and the availability of powerful parallel computers, make the Partitioned Adaptive Control a preferrable choice for real time applications in underwater environments. 


\section{REFERENCES}

[1] J.M. Walton, "Advanced Unmanned Search System", Proceedings of IEEE Oceans, pp. 13921399, 1991.

[2] D.N. Yoerger, J.E. Slotine, "Robust trajectory control of underwater vehicles", IEEE Journal of Oceanic Engineering, Vol. OE-10, No. 4, pp. 462-470, 1985.

[3] J. Yuh, "Modeling and Conirol of Underwater Robotic Vehicles", IEEE Transactions on Systems, Man and Cybernetics, Vol. 20, No. 6, 1990.

[4] H. Kazerooni, T.B. Sheridan, "Computer simulation and Control of Underwater Vehicles", M.I.T. Sea Grant College Program, MITSG 82-19, October 1982.

[5] M. Geruler, G.R. Hagen, "Standard equations of motion for submarine simulation", NSRDC Report 2510, June 1967.

[6] D.G. Lainiotis, "Partitioning: A Unifying Framework for Adaptive Systems, I-Estimation", Proccedings of IEEE, Vol. 64, No. 8, pp. 11261142, August 1976.

[7] D.G. Lainiotis, "Partitioning: A Unifying Framework for Adaptive Systems, II-Control", Proceedings of IEEE, Vol. 64, No. 8, pp. 11821198, August 1976.

[8] S.K. Katsikas, S.D. Likothanassis, D.G. Lainiotis, "On the Parallel Implementation of the linear Kalman and Lainiotis Filters and their efficiency", Signal Processing, No. 25, pp. 289305, December 1991.

[9] J. Deshpande, T.N. Upadhyay, D.G.Lainiotis, "Adaptive Control of linear stochastic systems", Automatica, Vol. 19, pp 107-115, 1973.

[10] K. Watanabe, ADAPTIVE ESTIMATION AND CONTROL : THE PARTITIONING APPROACH, Prentice Hall, 1992.

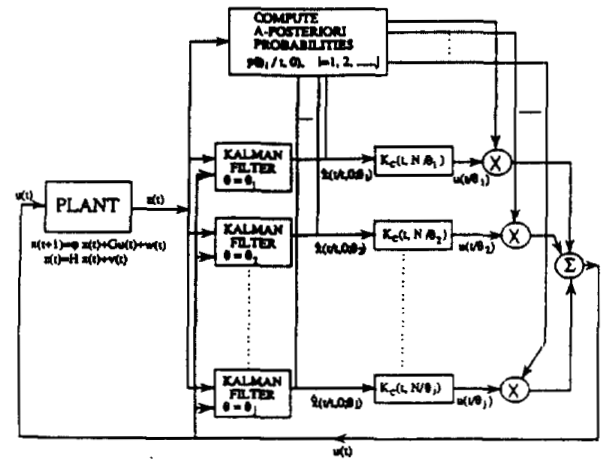

Fig. 1 PAC Structure

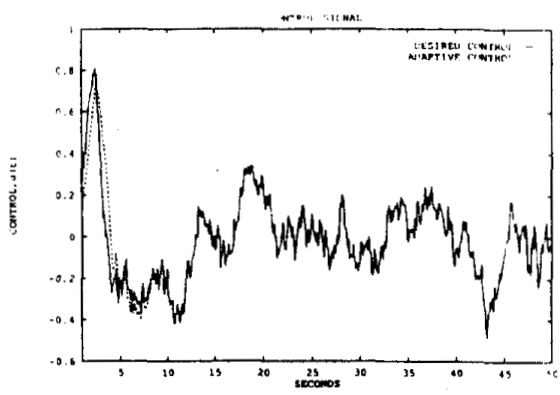

Fig. 2 AUV: Desired vs. Adaptive Control Signals

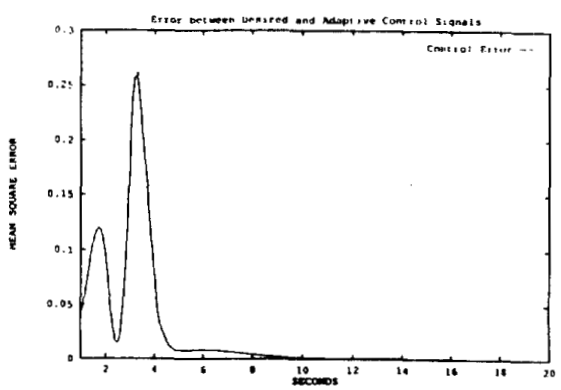

Fig. 3 AUV: Error between Desired and Adaptive Control Signals 


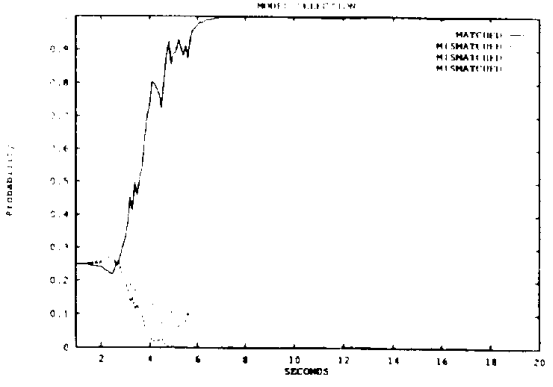

Fig. 4 Adaptive Filter: Model Selection

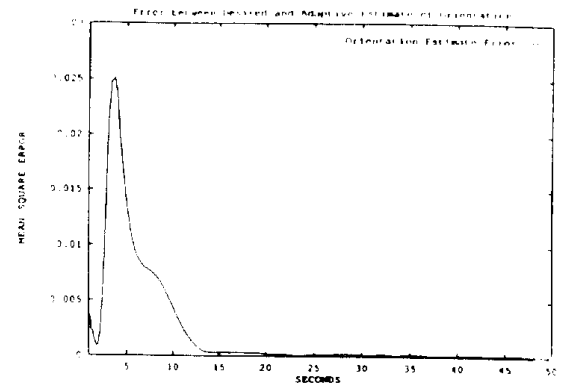

Fig. 6 AUV Orientation: Mean Square Error, $100 \mathrm{MCR}$

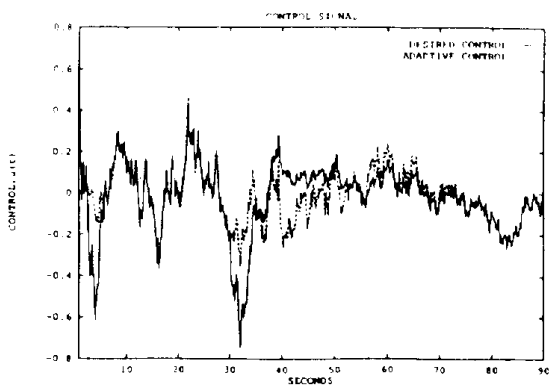

Fig. 8 AUV: Desired vs. Adaptive Control Signals

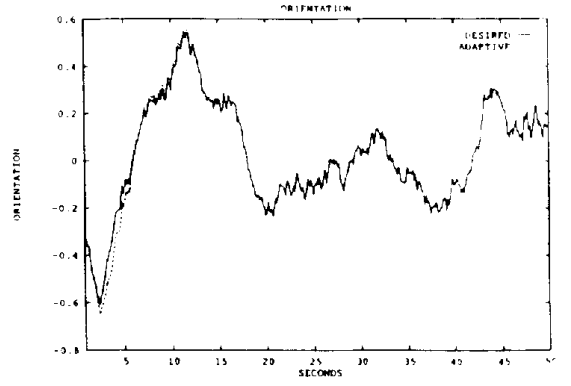

Fig. 5 AUV Orientation: Actual vs. Adaptive Estimate

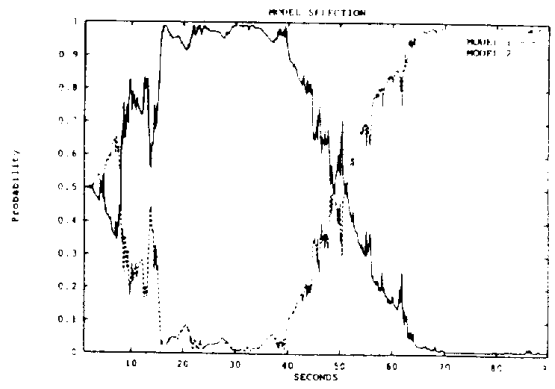

Fig. 7 Adaptive filter: Model Selection

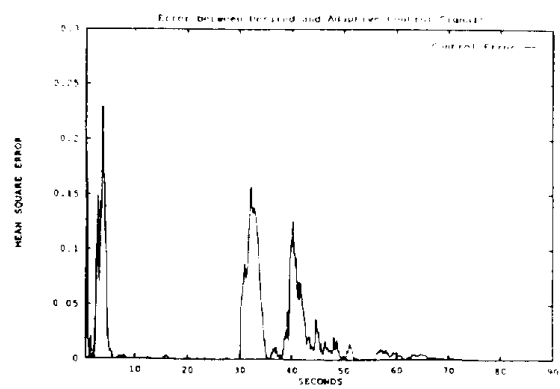

Fig. 9 AUV: Error between Desired and Adaptive Control Signals 


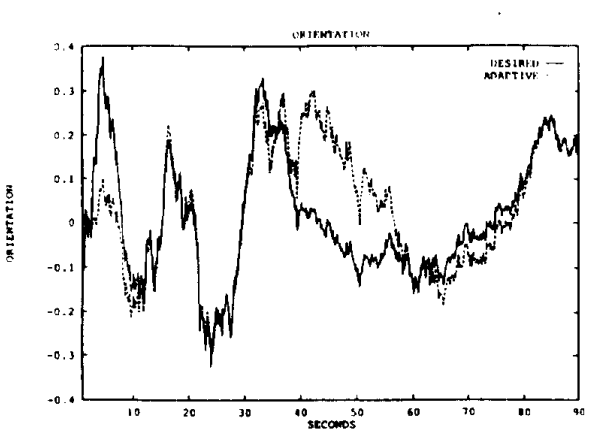

Fig. 10 AUV Orientation:Actual vs. Adaptive Estimate

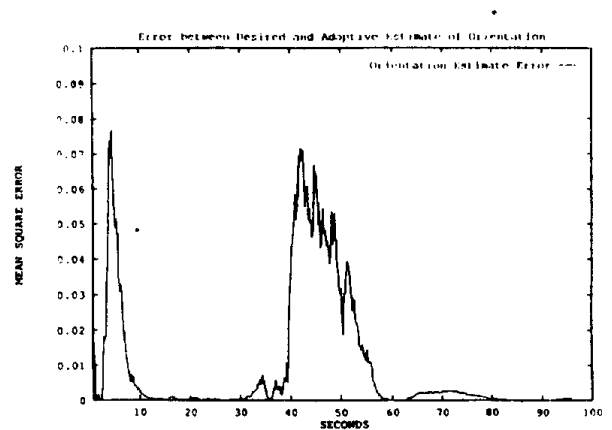

Fig. 11 AUV Orientation: Mean Square Error, $100 \mathrm{MCR}$ 